\title{
Nutrition, Vitamin B
}

National Cancer Institute

\section{Source}

National Cancer Institute. Nutrition, Vitamin B. NCI Thesaurus. Code C15927.

Role of Vitamin B, its precursons, and its analogs in cancer causation or prevention and in general health. 